\title{
V. Krystallographische Notizen über Baryumnitrat und Sphen.
}

\author{
Von \\ W. J. Lewis in London. \\ (Hierzu Tafe! IV, Fig. 1-4.)
}

\section{Baryumnitrat.}

Es war früher über das salpetersaure Baryum nur bekannt, dass dasselbe im regulären Syslem krystallisire, der parallelflächigen Hemiëdrie angehöre und die Formen (100), (111) und $\pi(210)$ zeige. Es haben jedoch schon die Beobachtungen von A. Scacchi (Poggend. Ann. d. Phys. 109, 366) und diejenigen von Baumhaur (d. Zeitschr. 1, 51) die Tetartoëdrie des Salzes wahrscheinlich gemacht; Ersterer beobachtete die tetartoëdrische Form $x \pi(124)$.

Im vorigen Herbst brachte mir Hr. Thom as Davies, vom britischen Museum, einen recht grossen und sehr flächenreichen Krystall, welchen er auf dem Boden einer Reagentienflasche ohne Etiquette fand, die viele Jahre lang unbenutzt auf der Seite gestanden hatte. Da der StöpseI mangelhaft geschlossen hatte, war allmählich die ganze Lösung verdampft und hat diesen einen Krystall geliefert. Ein an der Stelle, wo sich keine Krystallflächen befanden, losgetrenntes Stückchen liess erkennen, dass die Substanz $\mathrm{Ba}\left(\mathrm{NO}_{3}\right)_{2}$ sei. Die krystallographische Untersuchung zeigte nun mit Evidenz, dass dieses Salı te tartoëdrisch krystallisirt; es wurden folgende Formen beobachtet:

$$
\begin{aligned}
& a=(100) \quad \infty 000 \\
& t=x \pi(214)+\frac{402}{4} l \\
& n=x \pi(351)+\frac{50 \frac{5}{3}}{4} r \\
& h=x \pi(2 \pi 4)-\frac{402}{4} r
\end{aligned}
$$




$$
\begin{aligned}
& 0=x(\mid \bar{T} 1)-\frac{0}{2} \\
& l=x(311)+\frac{303}{2} \\
& s=x(2 \bar{T} 1)-\frac{202}{2} .
\end{aligned}
$$

Ein Blick auf die stereographische Projection Fig. 1, Taf. IV. zeigt, dass $t, n$ und $l$ nur in den abwechselnden (positiven) Oktanten, $h, o$ und $s$ nur in den negativen auftreten. Die Formen $t$ und $h$ entsprechen einander und bilden die parallelflächig hemiëdrische Geslalt eines Dyakisdodekaëders $\pi(124)=\left[\frac{402}{2}\right]$; jedoch zeigt die Verschiedenheit der physikalischen Beschaffenheit ihrer Flächen in benachbarten Oktanten deutlich die Telartoëdrie, indem diejenigen von $h$ gross und glatt, die von $t$ klein und rauh erscheinen. Die Flächen $n$ sind in den positiven Oktanten gross und glänzend entwickelt, während von den entsprechenden Flächen in den andern Oktanten keine Spur zu entdecken ist; ebenso treten auch die in gleicher Weise entwickelten Flächen o nur als negatives Tetraëder auf; $l$ ist gut ausgebildet und glänzend, $s$ sehr klein, aber recht glänzend; dieselbe Oberflächenbeschaffenheit zeigen auch die ziemlich gross entwickelten Würfelflächen.

Die hauptsächlichsten Zonen des Krystalls sind folgende : a $n l_{n,}, a_{\prime \prime} s_{, \prime}$, at, $h s_{,} h_{,}$, o $l_{n} n_{\prime \prime} h_{,}$. Diese waren bei der Bestimmung der Formen von erheblichem Nutzen. Die wichtigsten Winkel der Formen sind die folgenden :

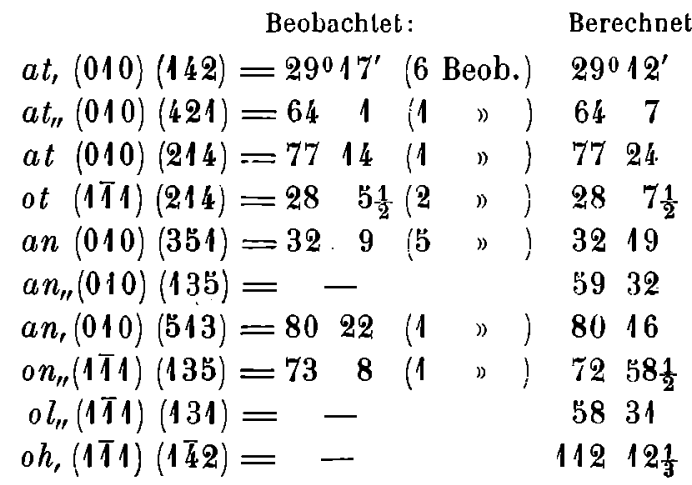

Die stereographische Projection, Fig. 1,

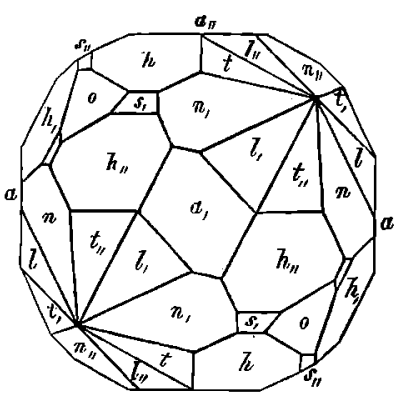
Taf. IV. giebt ein Bild der Zonen und der Vertheilung der Flächen, beistehender Holzschnitt ist eine orthogonale Projection auf eine Würfelfläche, während Fig. 2, Taf. IV. eine gewöhnliche schiefe Projection darstellt, in welcher der Einfachbeit wegen die kleinen Flächen $s$ ausgelassen sind. 
Der Krystall ist nach einer Richtung verlängert ; es scheint sich zuerst ein ovaler Ring gebildet zu haben, und auf diesem der Krystall gewachsen zu sein, so dass er mit seiner unebenen Unterfläche nicht am Boden der Flasche festsass; ebenso ist auch das obere Ende nicht vollkommen ausgebildet.

Der Krystall, auf das Polarisationsinstrument zwischen gekreuzte Nicols gebracht, depolarisirte das Licht. In monochromatischer Beleuchtung konnte keine bemerkbare Aenderung beobachtet werden beim Drehen des Krystalls, noch konnte das Licht ausgelöscht werden durch eine Drehung des Analysators. Weitere Versuche zur Entscheidung der Frage, ob in der That neben der anomalen Doppelbrechung noch eine Circularpolarisation vorhanden sei, konnten nicht angestellt werden, ohne den Krystall zu zerstören.

An einigen anderen Krystallen von Baryumnitrat, welche in wenigen Wochen aus einer Lösung auskrystallisirt waren, beobachtete ich die Formen :

$$
\begin{aligned}
& x(111)+\frac{o}{2} \\
& x(1 T 1)-\frac{o}{2} \\
& (101) \quad \infty O \\
& x(2 T 2)-\frac{2 O}{2} .
\end{aligned}
$$

Die Flächen des zweiten Tetraëders, dessen Combinationskanten mit (101) durch das Deltoiddodekaëder (2T2) abgestumpft waren, schienen glatter und glänzender zu sein als die des ersten.

\section{Sphen.}

An den Krystallen des Sphen findet man nicht selten zwei rauhe, schlecht entwickelte Flächen an der von $n=(\overline{1} 23)+\frac{2}{3} R 2$ und $c=$ (001) o $P$ gebildeten Ecke; sie besitzen oft das Ansehen unvollkommen angeschliffener Flächen. Die vollkommene Symmetrie ihrer Lage jedoch und auch ihre Häufigkeit beweisen, dass es wirkliche Krystallflächen sind. Eine ähnliche Form beobachtete Hessenberg (Mineralogische Notizen, VI, Fig. 34, deren Gopie Fig. 3, Taf. IV. darstellt) an den Krystallen vom Zillerthal, wo er sie stets mehr oder weniger deutlich entwickelt fand; er nahm jedoch an, dass diese rauhen Flächen nur eine Fortsetzung derjenigen von $n$ seien.

Unlängst erhielt ich nun einige Krystalle, an denen die fraglichen Flächen deutlich ausgebildet waren, und deren Form Fig. 4, Taf. IV. darstellt. Aber auch hier waren die erhaltenen Reflexe so schlecht, dass 
Messungen nur mit Sonnenlicht und nach leichtem Anölen der Flächen gewonnen werden konnten. Es ergab sich das Zeichen der Form $\varphi=$ $(\overline{3} .3 .10)+\frac{3}{10} P$, bezogen auf das in Miller's Mineral. angenommene Axenverhältniss.

Die Kantenwinkel sind folgende:

Beobachtel: Berechnet:

$\begin{array}{llll}n \varphi & 1102^{\prime} & 10^{\circ} 59^{\prime} \\ n_{1} \varphi & 3333 & 3319 \\ \varphi \varphi_{1} & 2233 \frac{1}{2} & 2231 \\ c \varphi & 2742 \frac{1}{2} & 2747\end{array}$

